\title{
Predicates or Formulas? Evidence from Ellipsis.
}

\author{
Irene Heim \\ Massachusetts Institute of Technology
}

\section{Syntactic structures and semantic types: some choices}

When we begin to construct a theory of semantic interpretation and the syntaxsemantics interface, we need to make a number of fundamental decisions that have no obvious empirical justification. Consider the following three questions, for each of which there are at least two answers.

First, what is the semantic type of VPs? One answer is that VPs are 1place predicates. An alternative answer is that VPs are formulas. ${ }^{1}$ For example, the LF-representation for John left on the "predicates" hypothesis may be (1).

[s John [vP left] ]

On the "formulas" hypothesis, the same sentence has an LF such as (2a) or (2b).
a. [S _ [vP John left] ]
b. [S John $n_{x}[v P x$ left] ]

In (2b), the subject is in its surface position and binds a variable in the VP, whereas in (2a) it has been reconstructed into the VP. If reconstruction of moved phrases is always an option in the derivation of LF, both structures are generated. The semantic interpretation of (1) and (2a) is straightforward, given suitable lexical entries and the rule of functional application. For structures like (2b), we need a semantic rule like (3). ${ }^{2}$

$$
\left[\left[\alpha_{x} \phi\right]\right]^{\mathrm{g}}=[[\phi]] \mathrm{g}[x /[[\alpha]] \mathrm{g}]
$$

Our second question is: what are the LF structures projected by quantificational determiners? Quantificational determiners are standardly taken to denote functions of type $<<e, t>,<<e, t>, t>>$. Given the functional application rule, they are therefore straightforwardly interpretable in the LF-structure (4), where $\zeta$ and $\xi$ are 1-place predicates.

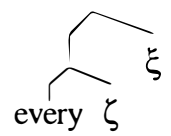


For example, the LF for every man left may look as in (5a) or (5b).
a. [s every man [vp left] ]
b. [s every man $\lambda \times[s \times[v P$ left] ] ]

(5b) would be the result of moving every man by $\mathrm{QR}$, where it is assumed that applications of QR leave a trace and automatically introduce a $\lambda$-abstractor right below the moved phrase. ${ }^{3}$ If $\mathrm{QR}$ is optional, both LFs are generated. Either way, every combines with two 1-place predicates. On the "predicates" hypothesis about quantificational determiners, this is the only option: quantificational determiners always appear in the configuration (4) with two 1-place predicates.

On an alternative view, quantificational determiners at LF carry a variable subscript and combine with two constituents $\phi$ and $\psi$ that are formulas, as in (6).

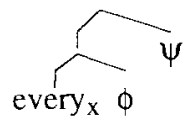

This "formulas" hypothesis goes along with the assumption that the NP after the determiner contains a silent subject, which is a variable coindexed with the determiner. It is also assumed that when a DP headed by a quantificational determiner moves, the trace it leaves is a variable coindexed with the determiner (and no $\lambda$-abstractor is introduced). The LF of our sentence every man left is then something like (7).

$$
\text { [ [DP every } \text { [NP } x \text { man] ] [ } x \text { left] ] }
$$

A suitable interpretation rule for such structures is (8). ${ }^{4}$

$$
\left[\left[\text { every }_{x} \phi \psi\right]\right]^{\mathrm{g}}=1 \text { iff }[[\psi]]^{\mathrm{g}[x / \mathrm{a}]}=1 \text { for every a such that }[[\phi]]^{\mathrm{g}[x / \mathrm{a}]}=1 \text {. }
$$

Third, what is the semantic type of restrictive relative clauses? Again, there is a "predicates" hypothesis and a "formulas" hypothesis. On the former, relative clauses are 1-place predicates. For instance, the relative clause which $t$ left has the LF-representation (9), where $x$ stands for the trace, and the moved which is represented and interpreted as a $\lambda$-abstractor.

$$
[\mathrm{CP} \lambda \times[s \times \text { left }]]
$$

Relative clauses then combine with the NPs they modify by the semantic operation of set-intersection. On the alternative view, relative clauses are formulas. The relative pronoun is itself a variable on this "formulas" hypothesis (like ordinary personal pronouns); it is not a $\lambda$-abstractor, nor is $\lambda$-abstraction 
introduced in its movement. It may or may not reconstruct at LF, so the LF for the relative clause which $t$ left is either (10a) or (10b).

(10) a. [CP - [s x left ] ]

b. [CP $x_{y}[s$ y left] ]

(10b) would be interpreted by rule (3). The semantic operation combining relative clause and NP is conjunction (presupposing again that the NP contains a subject).

To summarize, there are a number of cases where a kind of phrase might be semantically interpreted either as a 1-place predicate or as a formula, provided appropriate concomitant assumptions. Is there any point in arguing about which choice is right, or is it ultimately just a matter of taste, habit, and expository convenience?

The aim of this paper is to find evidence which favors the formulas hypothesis over the predicates hypothesis in each of the three cases we have looked at (VPs, arguments of determiners, relatives). The strategy I will employ is to test the two competing hypotheses in conjunction with a theory of VPellipsis. The empirical basis of my argument is a generalization about VP-ellipsis which was recently brought to light by Christopher Kennedy.

\section{Kennedy's generalization}

Kennedy (1994) observes and analyzes contrasts like the following. ${ }^{5}$

(11) I visited every country I had to. 'I visited every country I had to visit'

(12) I visited every town in every country I had to.

* 'I visited every town in every country I had to visit'

(11), with the reading indicated, is a run-of-the-mill grammatical case of antecedent-contained VP-deletion. (12), by contrast, is not acceptable on the indicated reading. 6 The relevant difference emerges when we look at the object of each instance of the verb visit in the following logical translations.

(13) $\forall x[x$ is a country \& I had to visit $x \rightarrow$ I visited $\mathrm{x}]$

(14) $\forall \mathrm{x}$ [x is a country \& I had to visit $\mathrm{x} \rightarrow \forall \mathrm{y}$ [y is a town in $\mathrm{x} \rightarrow$ I visited $\mathrm{y}]]$

In (13), which represents the good example (11), both instances of visit have the same object, namely the country-variable $x$. This is not so in (14), the rendition of 
the bad example (12). Here, the first visit (the one that represents the deleted verb) takes the country-variable $x$ as its object, whereas the second visit (corresponding to the verb in the overt antecedent) takes the town-variable $y$.

An analogous pattern, Kennedy argues, is seen in certain examples of VPdeletion which is not antecedent-contained. The grammatical (15) contrasts with the ungrammatical (16).

(15) Every man who wants to leave should. 'every man who wants to leave should leave'

(16) Every man who wants George to leave should.

* 'every man who wants George to leave should leave'

This time, please look at the subject of each verb leave in the logical translations.

$$
\forall \mathrm{x} \text { [ } \mathrm{x} \text { is a man \& } \mathrm{x} \text { wants } \mathrm{x} \text { to leave } \rightarrow \mathrm{x} \text { should leave] }
$$

$\forall \mathrm{x}$ [ $\mathrm{x}$ is a man \& $\mathrm{x}$ wants George to leave $\rightarrow \mathrm{x}$ should leave]

In the good case (17), the two instances of leave have the same subject, viz., the man-variable $x$. In the bad case (18), they have different subjects, George and $x$ respectively.

Both pairs of examples fall under a generalization which Kennedy (1994: 2) informally states as follows7: "Ellipsis between $\mathrm{VP}_{\alpha}$ and $\mathrm{VP}_{\beta}, \mathrm{VP}_{\beta}$ contained in an argument $A_{\alpha}$ of $\mathrm{VP}_{\alpha}$, is licensed only if $\mathrm{A}_{\alpha}$ is identical to the parallel argument $A_{\beta}$ of $V P_{\beta}$." (For there to be "ellipsis between" two VPs means that one is elided and the other its antecedent; Kennedy's formulation is deliberately neutral as to which of $\mathrm{VP}_{\alpha}$ and $\mathrm{VP}_{\beta}$ is the antecedent.) In (11) and (12), the antecedent VP's object contains the deleted VP. Hence the generalization demands "identity" between the two VPs' objects. In (15) and (16), the deleted VP's subject contains the antecedent VP. Thus the generalization demands "identity" between the two VPs' subjects. The relevant notion of identity is evidently not surface identity. It apparently has to make reference to semantic interpretation or to a semantically transparent syntactic level which in essential respects resembles the informal "logical translations" I employed above. Kennedy offers a concrete proposal in this regard, which I cannot discuss in the space of this paper. My own proposal will be developed over the course of the next sections. 


\section{A preliminary explanation}

To reiterate from the introduction, my strategy is to compare different conceptions of LF by testing their predictions in conjunction with a theory of ellipsis. I therefore must commit myself to some concrete assumptions about the principles governing ellipsis in general and VP-ellipsis in particular. For didactic reasons, I will at first adopt a theory which is simple and pleasing, but which cannot be exactly right, especially not in conjunction with the claims defended in this paper. This is essentially the classical theory of Sag (1976) and Williams (1977). It says, in a nutshell, that VP-ellipsis is grammatical iff the deleted VP and its antecedent have logically equivalent LF-representations. The relevant notion of logical equivalence is the standard one: two expressions $\alpha$ and $\beta$ are logically equivalent iff $[[\alpha]] g=[[\beta]] g$ for every variable assignment $g$.

Armed with this condition on ellipsis, let us take a closer look at the Kennedy examples. For each example, I will specify two different (sets of) LFs. First I will give the LF(s) that would be generated under the predicates hypotheses sketched in section 1. Then I give the LF(s) that the same example would have under the formulas hypotheses. As we will see, the logical equivalence condition on VP-ellipsis makes different empirical predictions depending on which choice of LF it is applied to. And in each case, the prediction based on the predicates hypotheses will be wrong, and the prediction based on the formulas hypotheses will be right.

Consider (15) and (16). Their LF-structures on the predicates hypotheses look as in (19) and (20) respectively. $\lambda x$ represents the relative pronoun, the first $x$ its trace, and the second $x$ in (19) the bound PRO. For your convenience, I have struck through the deleted VP and underlined the antecedent VP.

$$
\begin{aligned}
& \text { every }[\operatorname{man} \lambda x[x \text { wants } x \text { to }[v P \text { leave }]]][\text { should [vP teave }]] \\
& \text { every }[\operatorname{man} \lambda x[x \text { wants George to [vP leave }]][\text { should [vp teave }]
\end{aligned}
$$

Evidently, all concerned VPs denote the property [[leave]] and are thus logically equivalent. This implies that VP-ellipsis is licensed in both structures, and we do not predict that (16) should be any less acceptable than (15).

Now let's construct LFs for the same examples (15) and (16) which conform to the formulas hypotheses. One way to do this, at least, yields (21) and (22).

$$
\begin{aligned}
& \operatorname{every}_{x}\left[[\mathrm{x} \text { man }]\left[\mathrm{x} \text { wants } \_ \text {to }[\mathrm{VP} \underline{\mathrm{x} \text { leave }}]\right]\right][\text { should }[\mathrm{VP} * \text { leave }]] \\
& \text { every }_{\mathrm{x}}\left[[\mathrm{x} \text { man }]\left[\mathrm{x} \text { wants } \_ \text {to }[\mathrm{VP} \text { George leave }]\right]\right][\text { should }[\mathrm{VP} \times \text { leave }]]
\end{aligned}
$$


These were obtained by exercising the reconstruction option wherever possible; in particular, I have reconstructed $P R O$ and George into their VP-internal tracepositions. When we apply the logical equivalence condition on VP-ellipsis to these structures, we predict what we want to. In (21), both VPs are $x$ leave, hence they are equivalent. In (22), one VP is George leave and the other one is $x$ leave. There are many variable assignments under which these have distinct denotations. So we don't have logical equivalence, and ellipsis is not licensed - just as Kennedy observed.

The explanation is not complete before we have made sure that there isn't some other LF which can also be generated for (16) under the formulas hypotheses and in which the two VPs are equivalent. For example, what if we chose not to reconstruct George? And what if, moreover, we happened to put the same index on George and its trace as we put on every? Then we could generate an LF for the bad example (16) that looks as in (23).

$$
\text { every }_{x}[[x \text { man }][x \text { wants George } x \text { to }[x \text { leave }]]][\text { should }[x \text { teave }]]
$$

This alternative LF would license ellipsis under the equivalence condition and would thereby undermine the proposed explanation of Kennedy's contrast.

The problem we are encountering here turns out be an old one. Sag and Williams had it too (albeit in different kinds of examples ${ }^{8}$ ). Sag's remedy was to replace "logical equivalence" in his ellipsis licensing condition by a non-standard concept of "alphabetic variance." 9 I will do something very similar: keep the logical equivalence condition as it is, but add a general prohibition against "gratuitous" (i.e., semantically inert) coindexing. Here is a concrete formulation.

(24) No Meaningless Coindexing: If an LF contains an occurrence of a variable $v$ that is bound by a node $\alpha$, then all occurrences of $v$ in this LF must be bound by the same node $\alpha$.

For example, (23) is excluded by (24), since one occurrence of $x$ is bound by George $_{x}$ and the others are not. As far as I can see, (23) also excludes any other LFs for (16) that would conform to the formulas hypotheses while having logically equivalent VPs. So with the help of "No Meaningless Coindexing", the logical equivalence condition on VP-ellipsis in conjunction with the formulas hypotheses about LF-structure predicts the contrast between (15) and (16). But if the formulas hypotheses are replaced by the predicates hypotheses, then everything else being equal, this desirable prediction is lost.

Let us now turn to the other pair of examples, which involves antecedentcontainment. I begin again with the LFs that are generated under the predicates hypotheses. If we adopt "No Meaningless Coindexing" in conjunction with the predicates hypotheses, then any LFs we can generate for either (11) or (12) wind up failing the equivalence condition. They all look essentially like (25) and (26), 
with a different variable for each lambda. (Recall that $\lambda x$ represents the relative pronoun, and $\lambda y$ and $\lambda z$ result from applications of $\mathrm{QR}$.)

$$
\begin{aligned}
& \text { every }[\text { country } \lambda x[\operatorname{I~had~to~[VP~visit~} x]] \lambda y[\text { I PAST [VP visit } y]] \\
& \text { every [country } \lambda x[\operatorname{I~had~to~[vP~visit~} x]] \\
& \qquad \lambda y[\text { every [town in } y] \lambda z[\text { PAST [VP visit z }]]]
\end{aligned}
$$

visit $x$ is neither equivalent with visit $y$ nor with visit $z$. Accordingly, both examples should be ungrammatical.

Would it help not to adopt "No Meaningless Coindexing"? Apparently not. If we allow ourselves to reuse variables freely, we can (among others) generate the LFs in (27) and (28).

$$
\begin{aligned}
& \text { every [country } \lambda x[\operatorname{I~had~to~[VP~visit~} x]]] \lambda x[\text { I PAST [VP visit x }]] \\
& \text { every [country } \lambda x[\text { I had to [VP visit } x]]] \\
& \lambda y[\text { every [town in } y] \lambda x[\text { I PAST [VP visit } x \text { ]] }
\end{aligned}
$$

Now we succeed in licensing ellipsis in (27) under the equivalence condition; but we also license it in (28). Again, we do not predict Kennedy's contrast.

Under the "formulas" hypotheses, we can generate LFs for (11) and (12) that look as follows.

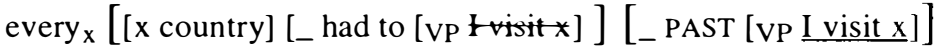

$$
\begin{aligned}
& \text { every }_{x}[[\mathrm{x} \text { country] [_ had to [VP I visit } \mathrm{x}]] \\
& \text { [every y [y town in } \mathrm{x}] \text { [_ PAST [VP I visit y]]] }
\end{aligned}
$$

These structures are promising. In (29), which represents the good example, we have equivalent VPs $I$ visit $x$ and $I$ visit $x$, and in (30), which represents the bad sentence, we have non-equivalent VPs $I$ visit $x$ and $I$ visit $y$. As before, of course, we must be aware that these are not the only LFs that are generated. In (29) and (30), I reconstructed everything as low as possible, which I need not have done. Had I exercised the reconstruction option more selectively, might I have been able to find an LF for the (bad!) example (12) which would have passed the equivalence condition after all? The situation turns out to be the same as it was with the previous pair of examples: Without the "No Meaningless Coindexing" condition, I would indeed be in trouble; but with its help, the potentially problematic alternatives to (30) are all unavailable. I invite the reader to check this. 
To summarize, we have been able to derive correct predictions about Kennedy's contrasts by combining three ingredients. The first two (essentially from Sag 1976) were the assumption that VP-deletion is licensed by logical equivalence, and the prohibition against meaningless coindexing. The third ingredient consisted in the formulas hypotheses regarding the LF-representations of VPs, relatives, and determiner quantification. This third ingredient was seen to be indispensable. The competing predicates hypotheses would not have supported the same desirable predictions. At least they would not have done so in conjunction with the same first two ingredients, nor did we see an obvious modification of those that would have helped. It seems, therefore, that we have come up with bona fide empirical evidence for the formulas hypotheses and against the predicates hypotheses.

This could have been the conclusion of the paper, were it not for a serious problem. The same package of assumptions which has served us so well in this section turns out to make terrible predictions for many other examples, including the most ordinary cases of VP-ellipsis.

\section{Ellipsis licensing and focus}

The assumptions that allowed us to explain Kennedy's contrasts make the unfortunate prediction that VP-ellipsis never allows non-coreferential subjects. This is blatantly false, of course. Consider (31).

(31) John called, and Mary did too.

'John called, and Mary called too'

On the formulas hypothesis about VPs, we cannot find a good LF for this example. If we reconstruct the subjects, we get (32a), if we don't reconstruct (but obey No Meaningless Coindexing), we get (32b).
a. _PAST [VP John call], and _ did [VP Mafy call] too
b. John $\mathrm{J}_{\mathrm{x}}$ PAST [VP $\left.\underline{\mathrm{x} \text { call }}\right]$, and Mary $\mathrm{y}$ did [VP $y$ catl] too

There is no way to meet the logical equivalence condition, and (31) is ruled out exactly like (16). The same problem arises if we try to extend the analysis of our (11) and (12) to Kennedy's original versions of these examples:

Polly visited every town Eric did.

(34) * Polly visited every town in every country Eric did. 
All LFs we can construct for the (good!) example (33) fail the equivalence condition because of the mismatch between Polly and Eric.

Clearly, at least one of the three ingredients in the current proposal must be wrong. Which one is it? I contend that the culprit is not the formulas hypothesis about VPs, but our out-dated assumptions about VP-ellipsis. I want to argue, in fact, that the problem we have run up against is nothing but an especially dramatic instance of a general type of problem which has already been known for a while to plague this classical theory.

A number of Sag's and Williams' recent critics have noted that their licensing conditions were too strict. ${ }^{10}$ Specifically, they systematically excluded sloppy identity readings for pronouns bound by something other than the antecedent VP's subject. Yet such readings are of ten available. A case in point is (35), from Jacobson (1992).

(35) Tom wanted Sue to water his plants, while John wanted Mary to.

'Tom 1 wanted Sue to water his 1 plants, while John 2 wanted Mary to water his 2 plants.'

In (my implementation of) Sag's theory, the problem with (35) presents itself as follows. In any LF that represents the intended sloppy reading and obeys the No Meaningless Coindexing convention, the two VPs, on the predicates hypothesis, will be water $x$ 's plants and water $y^{\prime}$ s plants, with distinct variables $x$ and $y .{ }^{11}$ These are not logically equivalent. (The formulas hypothesis about VPs would only make things worse here, of course.) So this sort of example shows that the classical theory of VP-ellipsis is too restrictive, quite independently of the choice between predicates and formulas which is the topic of my paper.

What is the remedy? A number of suggestions can be found in the literature, and this is not the occasion to compare them. I will follow an approach pioneered by Rooth (1992b), which proceeds by dividing the job of licensing VPellipsis between two separate conditions, of which one refers to the deleted VP itself and the other to a possibly larger phrase containing it. ${ }^{12}$ Ellipsis is licensed only when both conditions are met.

The VP-level condition requires, very roughly, that the deleted VP and its antecedent must be made up of the same lexical material. For instance, it would not allow matching a deleted VP of the form $x$ see Sue with an antecedent of the form $y$ be seen by John. But it doesn't care about matters of indexing. As far as this condition is concerned, $x$ see $y$ would qualify as an antecedent for $z$ see $u$. A precise and principled formulation of this condition, although ultimately very important for the credibility of my conclusions, is beyond the scope of the present paper. ${ }^{13}$

The second condition which Rooth proposes demands that the deleted VP has to be contained in a phrase which "contrasts appropriately" with some phrase that contains the antecedent VP. The relevant notion of appropriate contrast is the 
same one that characterizes all felicitous uses of contrastive focus. As far as this condition is concerned, VP-ellipsis is just a special case of so-called anaphoric deaccenting.

The following definition assumes familiarity with the basic concepts of Rooth's focus semantics. ${ }^{14}$

(36) A constituent $\phi$ contrasts appropriately with a constituent $\psi$ iff

(i) $\phi$ and $\psi$ don't overlap, and

(ii) for all assignments $\mathrm{g}$, the (regular) semantic value of $\psi$ w.r.t. $g$ is an element of the focus value of $\phi$ w.r.t. $g$.

For an illustration, consider a simple example of contrastive focus.

Mary called. Or perhaps JOHN called.

Capitalization of $\mathrm{JOHN}$ is meant to represent a pronunciation on which John bears the main stress of its sentence. ${ }^{15}$ Glossing over many non-trivial issues in the phonology and phonetics of focus, we assume that this prosody reflects a syntactic representation in which John is marked with the focus feature $F$ (and there is no other $F$ in the clause John called). By the rules for the compositional calculation of focus values, this implies that the focus value of the clause John $F$ called is the set of propositions $\{$ that $\mathrm{x}$ called: $\mathrm{x} \in \mathrm{D}\}$. The first clause of (37), Mary called, has as its (regular) semantic value the proposition that Mary called, which happens to be an element of this set. Therefore, John $F$ called contrasts appropriately with Mary called. (The non-overlap condition is also met; see more about the motivation for this in section 5 below.) Compare (37) to an infelicitous example.

\section{(38) John sent email. Or perhaps JOHN called.}

The main stress on John again determines the syntactic representation John $F$ called, with the same focus value as above. But in (38), there is no other constituent with which $J o h n_{F}$ called contrasts appropriately. The proposition denoted by the sentence John sent email is not in $\{$ that $\mathrm{x}$ called: $\mathrm{x} \in \mathrm{D}\}$.

Let us now analyze an example of VP-ellipsis, such as (35). The natural way to pronounce this example is with contrastive foci on John and Mary. So it seems to have the LF-representation in (39). (This structure is based on the predicates hypotheses; see below for the formulas alternative.)

(39) Tom $\lambda x[x$ wanted Sue to [VP water $x$ 's plants] $]$, while JohnF $\lambda y\left[y\right.$ wanted MaryF to [VP water $y^{\prime}$ 's plants]] 
On Rooth's two-level approach, the ellipsis is licensed only if, first, there is the appropriate lexical match between deleted VP and antecedent, and second, we can find a pair of containing phrases that meet the appropriate-contrast requirement. Since the first condition is oblivious to indexing choices, it is unproblematic in (39). What about the second condition? Suppose we choose the whole clause after while as the relevant containing phrase around the deleted VP. Now we must calculate the focus value of this phrase. This turns out to be the set $\{$ that $x$ wanted $y$ to water $x$ 's plants: $x, y \in D\}$. Can we find another phrase, surrounding the antecedent VP, whose regular value is in this set? Yes. The complete sentence before while will qualify, since it expresses the proposition that Tom wanted Sue to water Tom's plants. Thus we have shown how the example is licensed.

In this application of the appropriate-contrast condition, our choice of containing phrase was considerably larger than the deleted VP. Was this necessary, or could we have chosen a smaller phrase? Let's look at the other potential candidates. The smallest possible phrase containing the deleted VP is the deleted VP itself. Since this contains no focus, its focus value is the singleton set of its regular value. And since it contains an unbound variable not inside a focus, its focus value depends on the assignment. For any $\mathrm{g}$, it is the set containing just the property of watering $\mathrm{g}(y)$ 's plants. This being so, any phrase that the deleted VP appropriately contrasts with would have to denote this same property under each g. There is no other such phrase in (39), in particular not around the antecedent VP. So this candidate was "too small".

What about the infinitival clause MaryF to water y's plants? This phrase contains a focus and therefore has a non-trivial (multi-membered) focus value. But it still contains $y$ free, and its focus value still depends on the assignment. For any $\mathrm{g}$, it is the set of propositions \{that $\mathrm{x}$ waters $\mathrm{g}(y)$ 's plants: $\mathrm{x} \in \mathrm{D}$ \}. So this infinitival clause can contrast appropriately only with phrases $\alpha$ such that, for any $\mathrm{g}$, there is an $\mathrm{x} \in \mathrm{D}$ such that $[[\alpha]]^{\mathrm{g}}$ is the proposition that $\mathrm{x}$ waters $\mathrm{g}(y)$ 's plants. This cannot be unless $\alpha$ contains free $y$ as well. So we will find no such $\alpha$ around the antecedent VP in (39).

By the same reasoning, any larger phrase in which $y$ remains free is too small to satisfy the appropriate-contrast condition as it applies to the LF in (39). The minimal domain within which we can license the ellipsis is the $\lambda$-abstract $\lambda y . .$. (which was created by movement of the higher subject John, or maybe by the Derived VP Rule applied to the want-VP). So this is the kind of example which shows that ellipsis licensing must sometimes crucially refer to a domain larger than the elided VP, and which was therefore systematically troublesome for the classical theory.

To make the point I just did, I deliberately constructed the LF for (35) on the basis of the predicates hypotheses. It is important to see here that the insufficiency of the classical theory, and the motivation for a condition on genuinely larger phrases, is not contingent upon the formulas hypotheses for 
which I argue in this paper. Examples like (35) give us an independent reason to abandon the logical equivalence condition on VP-ellipsis and to explore alternatives such as the dual-level approach proposed by Rooth.

Rooth's approach is, in principle, compatible with either the predicates or the formulas hypotheses. We have just seen how it accounts for the ellipsis in (35) when combined with the predicates hypotheses. On the alternative formulas hypotheses, the analysis of the same example would be a little different, but equally straightforward. We could then generate an LF like (40).

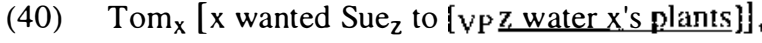
while [JohnF $]_{y}$ [y wanted [MaryF] to [VP water y's plantsis]

Again, the VP-level condition is met, since it doesn't care about the different variables in $z$ water $x$ 's plants and $u$ water $y$ 's plants. To satisfy the appropriatecontrast condition, we choose the entire clause after while. This has the focus value $\{$ that $x$ wants $y$ to water $x$ 's plants: $x, y \in D\}$, of which the regular value of the clause before while is an element.

Recall now the problem which stood at the beginning of this section: in conjunction with the classical logical equivalence condition on VP-ellipsis, the formulas hypothesis about VPs was incompatible with the grammaticality of (31), John called, and Mary did too. Let me show now that ellipsis is easily licensed in (31) if we combine the formulas hypothesis with the new two-level theory. Here is a suitable LF. Notice the focus on Mary, which is consistent with the phonetic evidence.

$$
\left.\left.\mathrm{John}_{\mathrm{x}} \text { PAST [VP } \underline{\mathrm{x} \text { call }]}\right] \text {, and [[MaryF] }\right]_{\mathrm{y}} \operatorname{did}[\mathrm{vP} \text { yeatl] }] \text { too }
$$

For the VP-level condition, $x$ call and $y$ call are a good enough match. (There would be a problem if the subjects were maximally reconstructed, as in (32a) above. But I haven't assumed reconstruction to be obligatory, and here I exploit this.) For the appropriate-contrast condition, choose the phrase [MaryF] did [VP $y$ calt]. Its focus value is $\{$ that $\mathrm{x}$ called: $\mathrm{x} \in \mathrm{D}\}$. So it contrasts appropriately with the first conjunct, $J o h n_{x} P A S T$ [ $V P \underline{x \text { call] }}$.

In sum, we have eliminated what seemed to be a fatal objection to the formulas hypothesis about VPs. On the best currently available theory of VPellipsis, this objection no longer applies. But what does that imply for the conclusions I had reached in the previous section? There, my arguments in favor of the formulas hypotheses had been based on the now rejected classical theory. Do they still go through now? 


\section{Kennedy's contrasts revisited}

My next goal is to show two things: first, that the Roothian two-level theory also supports an explanation of the Kennedy contrasts, and second, that this explanation also relies crucially on the formulas hypotheses as opposed to the predicates hypotheses. I begin again with examples (15) and (16).

(15) Every man who wants to leave should

(16) *Every man who wants George to leave should.

I take it that (15) has a focus on should, although for the time being, this assumption will play no role. On the formulas hypotheses, we can generate the following LF for it. (Except for the annotation of focus, this is exactly (21) above.)

$$
\text { every }_{x}[x \text { man } x \text { wants _to } \underline{x} \text { leave] [_ shouldF } * \text { leave] }
$$

The VP-level condition is obviously met. The appropriate-contrast condition is also easy to satisfy. In this case, even the deleted VP itself can qualify as the relevant containing phrase. Its focus value, for any $g$, is the unit set containing the proposition that $\mathrm{g}(x)$ leaves. The antecedent VP expresses that same proposition, for any g. So (15) is licensed, as it should be.

The task of showing that (16) is properly ruled out is a much more daunting one. Part of the complexity results from the fact that we must now consider LFs for every possible way of placing foci in (16). After all, the judgment regarding (16) (as reported by Kennedy), is that this sentence is unacceptable (on the intended reading) however you pronounce it. Hence a complete explanation of this data must cover all possible focus structures. How many of those are there altogether? This is probably not a simple question to answer, and luckily I will not have to get into it very deeply. Just one assumption is going to be crucial, namely, that there is a systematic incompatibility between focus and deletion. Specifically, deleted phrases or parts thereof cannot be Fmarked, nor can deletion affect a part of an F-marked phrase which would otherwise contain the strongest stress in that phrase. These laws are far from elementary or uncontroversial, of course. They should ultimately follow from the correct theory of focus phonology, but I am in no position to argue that they do. ${ }^{16}$

A further source of complexity is the optionality of reconstruction. In principle, there are thus many possible LFs for (16) to consider. However, we can deal with large groups of options at once. For one thing, any LF which, like (22) above, involves maximal reconstruction of the subject George, will not even make it past the VP-level licensing condition: 


$$
\text { every }_{x}[x \text { man } x \text { wants _ to George leave] [_ should * leave] }
$$

To avoid such mismatched lexical material inside the VPs, we must exploit the optionality of reconstruction. So let's turn to LFs which, up to possible differences in F-marking, look like (43).

$$
\text { every }_{x}[x \text { man } x \text { wants George } y \text { to y leave] [_ should * leave] }
$$

If these are to be ruled out, it must be due to the appropriate-contrast condition. Can we show that this condition must fail, whichever containing phrase around the deleted VP we choose, and wherever we place foci inside it? Consider the candidate phrases one by one. The smallest one is $x$ leave, the deleted VP itself. Being deleted, it cannot contain any focus, so its focus value for any g contains just the proposition that $\mathrm{g}(x)$ leaves. No phrase around the antecedent VP expresses this proposition. Next, include the should, which may or not be focussed. ${ }^{17}$ Suppose that it is. Then we are looking at the focus value of the phrase should $_{F} x$ leave, which is, for any $\mathrm{g}$, the set $\{\mathrm{f}($ that $\mathrm{g}(x)$ leaves): $\mathrm{f}$ is an alternative to the meaning of should $\}$. This set contains, besides the proposition that $\mathrm{g}(x)$ should leave, such propositions as that $\mathrm{g}(x)$ won't leave, that somebody wants $\mathrm{g}(x)$ to leave, that $\mathrm{g}(x)$ wants $\mathrm{g}(x)$ to leave, etcetera. ${ }^{18}$ None of these propositions is expressed by any constituent around the antecedent VP either. Clearly, if there were no focus on should, there would be even less of a chance of finding a suitable antecedent for appropriate contrast. Now the next larger phrase in (43) which contains the deleted VP is already the entire every-sentence. But since this includes the antecedent VP as well, we could not possibly find for it a non-overlapping phrase around the antecedent VP, as demanded by the first clause in my definition of appropriate contrast (see (36)). So I conclude that, at least as far as LFs of the form (43) are concerned, it is not possible to satisfy the appropriate-contrast condition and thereby license VP-deletion. I have still not given a real proof that the current theory generates no LF for (16) in which deletion could be licensed. But I have a plausible conjecture that this is the case. ${ }^{19}$

This is the occasion to comment on the "non-overlap" clause in my definition (36). ${ }^{20}$ Suppose I had omitted it, and suppose there are foci in (43) on both should and the DP headed by every. Then the focus value of the entire structure (43) presumably contains the proposition that George leaves, which is expressed by the embedded clause George to y leave. ${ }^{21}$ Thus ellipsis should be licensed in (16), contrary to fact. The prohibition against overlap is therefore an important ingredient of my analysis, and it would be desirable not to have to stipulate it, as I did in (36). One way or another, it should reduce to a general property of anaphora, given an appropriate understanding of the anaphoric nature of contrastive focus. Rooth (1992a), while he does not explicitly address the issue 
of overlap, has some pertinent remarks which suggest the possibilty of relating overlap to Condition C of the Binding Theory. ${ }^{22,23}$

Let us proceed to the contrasts in the antecedent-contained examples, such as $(11) /(12)$ and $(33) /(34)$.

(11) I visited every country I had to.

(12) *I visited every town in every country I had to.

(33) Polly visited every town Eric did.

(34) *Polly visited every town in every country Eric did.

Still sticking to the fomulas hypotheses, we begin again with the easier task of licensing the good examples. Here are some suitable LFs for (11) and (33), with Fs marking the audible foci on had and Eric.

$$
\begin{aligned}
& \text { every } \left._{x}\left[x \text { country }- \text { hadF }_{F} \text { to visit } x\right] \text { [_PAST } \underline{\text { visit } x}\right] \\
& \text { every }_{x}\left[x \text { town }\left[\text { Eric }_{F}\right]_{y} \text { did } y \text { visit } x\right]\left[\text { Polly }{ }_{z} \text { PAST } \underline{z \text { visit } x}\right. \text { ] }
\end{aligned}
$$

The VP-level condition is unproblematic in both cases, and the appropriatecontrast condition is also easy to meet: Choose the deleted VP itself in (44), whose (singleton) focus value of course contains the regular value of the (identical) antecedent VP. In (45), choose the slightly larger phrase [EricF $]_{y}$ did $y$ visit $x$. Since Eric is focussed, the focus value of this phrase, for any g, is the set $\{$ that $\mathrm{y}$ visited $\mathrm{g}(x): \mathrm{y} \in \mathrm{D}\}$. It thus contrasts appropriately with the phrase Polly $y_{z}$ PAST $z$ visit $x$.

We proceed to the harder task of ruling out the bad cases. The following potential LF-schemata (leaving open where the $F$ s are) are okay by the VP-level condition.

$$
\begin{aligned}
& \text { every }_{x} \text { [ } x \text { country _ had to I visit } x \text { ] } \\
& \text { [every } y \text { [y town in } x \text { ] [_ PAST I visit } y \text { ]] }
\end{aligned}
$$

What would it take to satisfy the appropriate-contrast condition in (46) or (47)? The candidates for larger phrases around the deleted VP are (i) I visit $x$, (ii) had to $I$ visit $x$, (iii) $x$ country _had to I visit $x$, and (iv) every $y_{x}$ [ $x$ country _ had to I visit $x$ ] in (46) (and the analogous ones in (47)). The next larger phrase would be the complete sentence, which is unavailable because of the overlap prohibition. 
We may safely disregard (iv), since it is of a semantic type unlike any phrase around the antecedent VP. As for the other three, let's maximize our chances of finding an appropriate contrast by positing foci wherever possible. This means an $F$ on had and an $F$ on $x$ country (and in (47), an $F$ on Eric). Even so, we won't succeed. All the focus values of these phrases depend on $g$. The focus value of (i) I visit $x$ contains only the proposition that I visit $\mathrm{g}(x)$. The focus value of (ii) had $_{F}$ to I visit $x$ contains various modalizations of this proposition. The focus value of (iii) $[x \text { country }]_{F}$ had $_{F}$ to I visit $x$ contains conjunctions of these modalizations with something else. But the constituents around the antecedent VP in (46) do not express any propositions in these sets. They instead talk about my visiting $\mathrm{g}(y)$, or about my visting every town in $\mathrm{g}(x)$. Analogous observations apply in the case of (47). This was only a sketchy survey, of course, but I claim that no genuine alternatives arise from other derivable LFs, and that the ellipsis in (12) and (34) is properly ruled out by the appropriate-contrast condition.

Where do we stand with respect to the goals of this section? I have argued that, given the formulas hypotheses about LF-structure, the Roothian two-level approach to ellipsis-licensing can correctly predict Kennedy's contrasts. This is a nice result in itself, but not the central claim of this paper. My main goal is to distinguish the formulas hypotheses from the predicate hypotheses. So the question I must now address is whether the predicate hypotheses would be able to support an equally successful Roothian account of Kennedy's contrasts. I will answer this question in the negative. Specifically, I will show that the appropriate-contrast condition no longer suffices to rule out the bad examples (16), (12), and (34), if we allow them to have the sorts of LFs that are generated under the predicates hypotheses.

Look at the following predicates-style LF for (16).

every [man $\lambda x[x$ wants George to leave] $][$ should $F$ leave]

The VP-level condition is evidently met, and there is more than one way of meeting the appropriate-contrast condition as well. The simplest way is to choose the deleted VP itself as the containing phrase. Its focus value is the singleton of the property of leaving, which is also the property expressed by the antecedent VP. At this point, we might consider adopting a slightly more restrictive definition of "appropriate contrast", which would require (in addition to the existing clauses of (36)) that the phrase $\phi$ contain at least one focus. ${ }^{24}$ This would disqualify the deleted VP itself as a candidate, and we would have to find a genuinely larger phrase in (48) to satisfy the appropriate-contrast condition. But then we only need to choose the phrase should $_{F}$ leave. should here is interpreted as a function from properties to properties, so the natural alternatives to its denotation will be other functions of this type. Now consider the function $\mathbf{f}$ which maps any property $\mathrm{P}$ to the property of wanting George to have $\mathrm{P}$. If $\mathbf{f}$ is among the alternatives to $[[$ should $]]$, then should $F$ leave contrasts appropriately with the 
VP want George to leave in (48). For notice that this VP denotes $\mathbf{f}([[$ leave $]])$. So the ellipsis should still be licensed. I conclude that the more restrictive definition of "appropriate contrast" is not sufficient to prevent ellipsis licensing in (48).

Of course, if we want to hold on to the predicates hypotheses and still account for Kennedy's facts, we might entertain other amendments to the theory of ellipsis or focus. Maybe the function $\mathbf{f}$ just doesn't qualify as an alternative to the meaning of should. But why not? There may be a principled answer to this question, but for the time being, I am not aware of one. 25

Let us now look at some LFs which the predicates hypotheses provide for the antecedent-contained unacceptable examples, (12) and (34).

$$
\begin{aligned}
& \text { every }\left[\text { country } \lambda x\left[\operatorname{I~had}_{F} \text { to visit } x\right]\right] \\
& \lambda y[\text { every }[\text { town in } y] \lambda z[\text { I PAST visit } z]] \\
& \text { every }\left[\text { country } \lambda x\left[\text { Eric }_{F} \text { did visit } x\right]\right] \\
& \lambda y[\text { every }[\text { town in } y] \lambda z[\text { Polly PAST visit } z]]
\end{aligned}
$$

Again, there is clearly no problem with the VP-level condition. What about the appropriate-contrast condition? In (50), it is particularly easy to meet: just choose the relative clause surrounding the deleted VP, viz., $\lambda x$ [Eric $F$ did visit $x$ ]. Because of the focus on Eric, its focus value is the set of properties \{having been visited by $\mathrm{x}: \mathrm{x} \in \mathrm{D}$ ]. The phrase $\lambda z$ [Polly PAST visit $z$ ] (which is the $\lambda$-abstract resulting from the application of $\mathrm{QR}$ to the 'town'-quantifier) evidently denotes a property in this set. (49) is not much different. Here too, the relative clause around the deleted VP $\left(\lambda x\left[I h_{F}\right.\right.$ to visit $\left.\left.x\right]\right)$ turns out to contrast appropriately with the phrase $\lambda z$ [I PAST visit $z$ ], assuming that the meaning of the past tense operator is an alternative to the meaning of the modal have to. (Note that we can hardly avoid this assumption if we don't want to rule out the good example (11) as well.)

I conclude, then, for the second and final time, that the formulas hypotheses are superior to the predicates hypotheses. In conjunction with what, to the best of my current knowledge, is the correct theory of VP-ellipsis, the formulas hypotheses support an explanation of the Kennedy contrasts, while the predicates hypotheses do not.

\section{Argument-contained ellipsis and focus: further empirical issues}

The gist of my analysis of Kennedy's unacceptable cases is that they fail because their structure somehow "leaves no room" for the contrastive focus that would save them. In ordinary examples like (31) John called, and Mary did too, the deleted VP is allowed to have a different subject, because that subject can be 
focussed, and its focus can be interpreted in a domain which is separate from the antecedent VP. But in Kennedy's (16) *Every man who wants George to leave should, the same thing is not possible. If we place a focus on the whole quantifier every man who wants George to leave, that focus cannot be interpreted in a domain excluding the antecedent VP. And if we try to place the focus on a lower subject position, i.e., on a variable bound by this quantifier, it will wind up on a trace, where it cannot be phonetically realized.

If this is basically the right diagnosis of the phenomenon, we expect to find other kinds of cases where a VP cannot delete simply because it lacks a subject which is both phonetically overt and sufficiently local. Indeed, we find this situation in coordination structures where the two conjuncts share a single overt subject. ${ }^{26}$ Compare (51) to (52), which differs minimally by the presence of an overt and stressed pronoun.

*Sue wants George to leave, but should.

\section{Sue wants George to leave, but SHE should.}

Interestingly, this type of example does not fall under the generalization which I quoted from Kennedy. Neither VP in (51) is contained in the other's subject. However, (51) does have this in common with (16): the smallest constituent which contains the deleted VP and an overt binder of its subject-position also contains the antecedent VP. It is this containment relation which really causes the ungrammaticality, if my analysis is on the right track.

The following pair is also instructive.

*Every man who wanted Mary to leave promised to.

Every man who wanted Mary to leave promised HE would.

Whether (53) falls under Kennedy's generalization depends. If you assume that there is no subject position in the to-infinitive, and if you count the every-DP as an argument of both leave and promise, then (53) is covered. But if (53) exhibits essentially the same structure and argument-relationships as (54), except that it has an empty pronoun ( $P R O)$ where (54) has an overt one, then it is not. From the perspective of the analysis pursued in this paper, the ungrammaticality of (53) is expected independently of this decision. What matters is that the to-infinitive lacks a subject that bears focus.

We further predict - correctly, I think - that variants of (52) and (54) with an overt, but completely deaccented, pronoun are as unacceptable as (51) and (53). This too indicates that argument-containment in the sense of Kennedy's generalization is not always necessary to produce the violation. 
The focus-based approach I am advocating might also be expected to throw light on the fact that at least some speakers can improve the bad examples by adding 'too', 'instead', or an adverbial reflexive. Kennedy (1994: 4, note 3) reports that some of his informants find (55) better than the corresponding sentence without instead, and I have heard people volunteer (56) (though others rejected it).

(55) The woman who thought the man from Portland would catch a tuna did instead.

Every man who wants George to leave should himself.

It appears that the added item in these examples serves to host a contrastive focus. This is suggestive, but I don't have an analysis of what exactly is going on here.

There are other loose ends, and some open problems may well be very serious. While Kennedy's generalization treats the deleted VP and its antecedent in a completely symmetrical fashion, my approach, following Rooth (1992b), does not. For ellipsis to be licensed, I have required that some phrase around the deleted VP must contrast appropriately with a phrase around the antecedent. But I have not required that there also be a phrase around the antecedent VP which contrasts appropriately with one around the deleted VP. As a consequence of this, I actually do not rule out the following variant of (16), in which it is the first VP that is deleted. 27

(57) Every man who wants George $\mathrm{F}$ to should leave.

Provided that George is focussed, the appropriate-contrast condition can be satisfied in the following LF for (57). Note that the focus value of [George $F]_{y}$ to $y$ leave contains the regular value of $x$ leave (for any $\mathrm{g}$ ).

$$
\left[\text { every }_{x}\left[[\mathrm{x} \text { man }]\left[\mathrm{x} \text { wants }[\text { George }]_{\mathrm{y}} \text { to } y \text { teave }\right]\right]\right][\text { should } \underline{x \text { leave }}]
$$

But according to Kennedy (and at least some of the other native speakers at the conference), (57) is no better than (16). I am not ready to defend a solution to this problem. Perhaps it can be argued that cases of backward ellipsis are generally awkward unless there is mutual contrast between phrases around the deleted and overt VPs. Obviously, this would require further empirical investigation and analysis. 


\section{Conclusion}

Suppose the various loose ends can be tied, and my explanation of Kennedy's contrasts essentially holds up. Then what have we learned, and what questions should we ask next? So far, I have only stated my conclusions in rather particular terms: VPs are formulas. Relative clauses are formulas. The sister nodes of determiners and moved DPs are formulas. This is a little unrevealing. For one thing, I should probably avoid the term "VP". The recent syntactic literature has entertained various highly articulated structures in the verbal-inflectional domain, and what is called a "VP" there is of ten quite small and expressly excludes the verb's external argument. What I have been talking about here is really the domain which is affected by so-called VP-ellipsis, whatever category that may be. Even this is not quite accurate. What does "affected by VP-ellipsis" mean within the two-level approach to ellipsis licensing? If you think about the logic of my argumentation, it turns out that the relevant domain is the smallest phrase which could potentially satisfy the appropriate-contrast condition. This phrase, I have shown, has to be a formula rather than a predicate. Now, I have been assuming that the smallest possible domain for satisfaction of the appropriate-contrast condition is the same constituent which is referred to by the "VP-level condition" (the condition requiring lexical identity), and also that this is also the same constituent which is affected by phonetic deletion. But if one or both of these assumptions will have to be revised, then it is important to be aware that my conclusion regarding "VPs" applies only to the domain relevant to the appropriate-contrast condition. 28

This leads to a more general question about the proper statement of my conclusions. Can I really claim that the various predicates generated by the predicates hypotheses don't exist, or might it be that the appropriate-contrast condition just doesn't see them? Strictly speaking, I have at best shown the latter. It could turn out that the predicates hypotheses are correct after all, and that it is a special consequence of the theory of contrastive focus that only formula-sized constituents can enter into contrast relations. The Kennedy paradigm then could receive essentially the same explanation, but nothing would follow beyond the domain of focus and ellipsis theory. (To appreciate that this is a viable alternative, notice that even on the predicates hypotheses, LFs contain plenty of formulas too. The objections I raised against the predicates hypotheses did not turn on a lack of formulas but on an excess of predicates.) This possibility certainly remains open. But the stronger hypothesis is prima facie more interesting: the reason why the appropriate-contrast condition can never be met by a pair of predicates is simply that no such predicates exist. Evidently, we would hope to eventually find confirmation for this claim from other, superficially unrelated, domains of evidence.

And while we search for such evidence, we want to theorize about the general principles of syntax and semantics which are ultimately responsible for 
the fact that we found formulas rather than predicates where we did. What have to be our basic assumptions about the universal inventory of semantic composition operations, about possible meanings of lexical items, about the construction of phrase structure, and about the nature of movement, so that the theory makes available just those LF-structures which we found to be attested?

\section{Endnotes}

*Earlier versions of this material were presented in seminars and colloquia at UMass Amherst, USC, M.I.T., and UT Austin, at the conference "Sinn und Bedeutung" in Tübingen and, of course, at SALT 7. I would like to thank the organizers and audiences of all these presentations for their interest and encouragement. I especially benefitted from comments and questions by Danny Fox, Dan Hardt, Jim Higginbotham, Polly Jacobson, Chris Kennedy, Mats Rooth, and Barry Schein. About many of these questions and comments, I still need to think a little longer. So please don't be disappointed if I have not yet addressed them properly.

'I use "formula" in the sense of standard treatments of Predicate Logic. A formula (unlike a "sentence") may contain free variables, but given an assignment of values to the variables, it has the same type of denotation as a sentence, i.e., a truth-value (in an extensional semantics) or a proposition (in an intensional semantics).

${ }^{2} \mathrm{~g}[x / \mathrm{a}]$ is that variable assignment which maps $x$ to a and is otherwise just like $\mathrm{g}$. ${ }^{3}$ For this conception of $\mathrm{QR}$, as well as for other background assumptions regarding the syntax-semantics interface, see Heim and Kratzer (in press).

${ }^{4}$ This is actually just an ad hoc syncategorematic version of a special case of the rule. For a general formulation, we need a semantic framework in which semantic values are functions from variable assignments. Such a framework is needed anyway for the compositional calculation of focus values (see Rooth 1985).

5 (11) and (12) are based on Kennedy's (3a,b) (which appear as (33), (34) below). The reason why I do not use the original examples here will soon become apparent. (15) and (16) below differ only trivially from Kennedy's (15), (16).

${ }^{6} \mathrm{It}$ is not relevant here whether the sentence is acceptable on another antecedentcontained reading, viz., ' I visited every town in every country I had to visit every town in'. That would not be a counterexample to Kennedy's generalization.

${ }^{7}$ The reader is urged to consult Kennedy's paper for a fuller range of examples and a thorough defense of the generalization's descriptive correctness.

${ }^{8}$ For example, the logical equivalence condition by itself does not predict that the sentence First John saw his mother, and then Bill did cannot mean that John saw Fred's mother and Bill saw his own mother. It would wrongly license deletion in an LF like (i), in which the first pronoun is free and the second one bound. 
It might be thought that this problem disappears if referential pronouns are not analyzed as free variables (but, e.g., as indexicals). However, this is not the solution, because the same problem arises in more complicated cases where referential readings are not at issue. For instance, Every boy said that first John saw his mother and then Bill did cannot mean that every boy $\mathrm{x}$ said that John saw $x$ 's mother and Bill his own.

${ }^{9}$ On the standard definition, alphabetic variance is a relation between (types of) expressions, and identical expressions are ipso facto alphabetic variants. See, e.g., Kalish, Montague and Mar (1980: 346ff.). Sag's notion is crucially a relation between occurrences of expressions, and defined in such a way that two occurrences of the same expression (e.g., the two VPs in (i) of the previous note) are not necessarily "alphabetic variants". See Sag (1976: 104ff.).

${ }^{10}$ For an overview, see Fiengo and May (1994) and the references cited there.

${ }^{11} \mathrm{Or}$, if Partee's Derived VP Rule (Partee 1975) is applied, they will be $\lambda z . z$ water $x^{\prime}$ s plants and $\lambda u$. $u$ water $y$ 's plants, which are likewise non-equivalent.

${ }^{12}$ See Tancredi (1992) for an independent related approach, and recent work by Tomioka (1997) and Wold (in prep.) for discussion and extensions of Rooth's proposal.

13Rooth's (1992b) observations and discussion go a long way towards sorting out the relevant empirical issues and delineating the role of this VP-level condition in the overall theory. He does not attempt an explicit formulation, however. For commentary and an interesting solution to the problems left open by Rooth, see Wold (in prep.).

${ }^{14}$ See Rooth (1985).

${ }^{15}$ The prosody and focus structure of the first sentence (Mary called) is left open here. There may or may not be an "anticipatory" contrastive focus on Mary in addition to the "retrospective" contrastive focus on John. See Rooth (1992a).

${ }^{16}$ There does not seem to be a lot of literature that develops the phonological side of Rooth's focus theory. Notable exceptions are Truckenbrodt (1995) and Rooth (1996b).

${ }^{17} \mathrm{~A}$ focus on the whole constituent should $x$ leave is impossible, I take it, because it would have to be realized by a main stress in the deletion site.

${ }^{18} \mathrm{We}$ do not want to assume that the focus value of should $_{F} x$ leave is the entire set $\left\{\mathrm{f}\left(\right.\right.$ that $\mathrm{g}(x)$ leaves): $\left.\mathrm{f} \in \mathrm{D}_{<<\mathrm{s}, \mathrm{t}>,<\mathrm{s}, \mathrm{t}>>}\right\}$, since this would be the set of all propositions whatsoever. There has to be some restriction to those functions in $\mathrm{D}_{<<\mathrm{s}, \mathrm{t}>,<\mathrm{s}, \mathrm{l}\rangle>}$ which are natural alternatives to [[should $]$.

${ }^{19}$ Another class of LFs to consider are those which differ from (43) by an additional (unreconstructed) intermediate trace of the every-DP:

$$
\text { every }_{x}\left[x \text { man } x \text { wants George } y \text { to } y \text { leave] } \left[x_{z} \text { should } z\right.\right. \text { leave] }
$$


However, since a trace cannot be focussed any more than other phonetically missing constituents, the phrase $x_{z}$ should $z$ leave can have a focus at most on should. Its focus value thus coincides with that of should $_{F} x$ leave, which we have already considered.

${ }^{20}$ The potential problem I am about to describe, and hence the need for the nonoverlap clause, was brought to my attention by Danny Fox and by Barry Schein.

${ }^{21}$ I am glossing over many technical details here. I have not actually shown how to calculate regular semantic values, much less focus values, for quantificational DPs as represented under the formulas hypothesis. (See note 4.) But however this is done in detail, we will want to license contrast in otherwise similar cases where there is no overlap, e.g. George left, and [every doctor $]_{F}$ did too.

${ }^{22}$ In the present paper, I have abstracted away from too many syntactic and semantic details of Rooth's original proposal to be more specific. In a nutshell, Rooth assumes that contrast is represented syntactically by coindexing of a covert focus anaphor with an antecedent phrase. And if the c-command domain of the focus anaphor is the scope of the focus interpretation operator whose argument it is, then the sort of overlap I need to exclude involves c-command by the focus anaphor of its antecedent. See Rooth (1992a: 87, note 8).

${ }^{23}$ The role which is played by the non-overlap condition in my proposal is at least vaguely reminiscent of a central feature of Kennedy's own analysis of his contrasts. Kennedy (1994) relates the unacceptability of (16), (12) etc. to a constraint against "referential circularity". This may indicate that the two analyses are not as different as they superficially appear. A careful comparison should be instructive, but is beyond the scope of this paper.

${ }^{24}$ This requirement looks a bit arbitrary in the context of my abbreviated rendition of Rooth's analysis of contrastive focus. But it is natural or even unavoidable in the context of certain recent versions of Rooth's general theory of focus interpretation. In the framework of Wold (1996), it amounts to a special case of the prohibition against vacuous variable binders, and in the movement theory entertained in Rooth (1996a), it follows automatically from the identification of the F-feature with the focus-interpretation operator. In a different way, it also follows from Truckenbrodt's (1995) principle of maximizing the domain of focus interpretation. There seems to be empirical evidence for it as well, although the relevant argumentation is complex. (See Truckenbrodt (1995) for pertinent discussion.) For the purposes of the present paper, I remain agnostic about it. If there is independent reason to assume it, then the correct analysis of examples (15) and (11) cannot be exactly as I gave it earlier in this section. In (42) and (44), the appropriate-contrast condition could then not be fulfilled by the deleted VPs themselves. But notice that it would still be satisfied by the phrases should $F$ $x$ leave in (42) and hadF $_{F}$ to I visit $x$ in (44). So we need not worry.

${ }^{25}$ It may be observed that there is the following difference between [[should]] and f. Whereas [[should ]], the property operator, derives by a standard type-shift 
operation from a propositional operator, there is no corresponding type$<<s, t>,<s, t>>$ source for $\mathbf{f}$. But why should the alternatives to [[should ]] be constrained to property operators derived from propositional operators? Isn't the only really principled answer to this question that should itself denotes the basic propositional operator in the first place, as it would have to under the formulas hypothesis about VPs?

26I need not take a stand here on whether this is IP conjunction with a null subject in the second Spec of IP, or just I-bar conjunction. Either way, there is no focusable spec of IP in the second conjunct.

${ }^{27}$ This was pointed out at the conference by Dan Hardt.

${ }^{28}$ For instance, suppose we adopt the assumption that appropriate contrast is defined only for phrases which contain at least one focus. (I briefly considered this in section 5 and note 24.) Then the smallest phrase which could possibly satisfy the appropriate-contrast condition will always have to be at least the node right above the overt auxiliary. In that event, the argumentation I have offered shows, strictly speaking, merely that this phrase is a formula, not that any proper part of it is. However, on most views of syntactic structure, the mother node of the auxiliary won't dominate a subject unless that subject is already in the auxiliary's complement.

\section{References}

Fiengo, Robert and Robert May (1994) Indices and Identity, M.I.T. Press: Cambridge.

Heim, Irene and Angelika Kratzer (in press) Semantics in Generative Grammar, Blackwell: Oxford.

Jacobson, Pauline (1992) "Antecedent-Contained Deletion in a Variable-Free Semantics," in Chris Barker and David Dowty (eds.) SALT 2, Working Papers in Linguistics, no. 40, Ohio State University, Columbus, 193-213.

Kalish, Donald, Richard Montague and Gary Mar (1980) Logic. Techniques of Formal Reasoning, 2nd edition, Hartcourt Brace Jovanovich: New York.

Kennedy, Christopher (1994) "Argument Contained Ellipsis," Linguistics Research Center report series, Cowell College, UCSC, Santa Cruz.

Partee, Barbara (1975) "Montague Grammar and Transformational Grammar," Lingusitic Inquiry 4, 203-300.

Rooth, Mats (1985) Association with Focus, Univ. of Massachusetts Ph.D. thesis, distributed by GLSA, Amherst.

— (1992a) "A Theory of Focus Interpretation," Natural Language Semantics 1, $75-116$.

- (1992b) "Ellipsis Redundancy and Reduction Redundancy," in Steve Berman and Arild Hestvik (eds.) Proceedings of the Stuttgart Fllipsis Workshop, 
report series Sprachtheoretische Grundiagen für die Computerlinguistik, no. 29, SFB 340, IBM Heidelberg.

- (1996a) "Focus," in Shalom Lappin (ed.) The Handbook of Contemporary Semantic Theory, Blackwell: Oxford, 271-297.

- (1996b) "On the Interface Principles for Intonational Focus," in Teresa Galloway and Justin Spence (eds.) SALT 6, CLC Publications, Cornell Univ., Ithaca, 202-226.

Sag, Ivan (1976) Deletion and Logical Form, M.I.T. Ph.D. thesis; published in 1980 by Garland Press: New York.

Tancredi, Christopher (1992) Deletion, Deaccenting, and Presupposition, M.I.T. Ph.D. thesis, distributed by MITWPL, Cambridge.

Tomioka, Satoshi (1997) Focusing Effects in VP-Ellipsis and Noun Phrase Interpretation, Univ. of Massachusetts Ph.D. thesis.

Truckenbrodt, Hubert (1995) Phonological Phrases: Their Relation to Syntax. Focus and Prominence, M.I.T. Ph.D. thesis.

Williams, Edwin (1977) "Discourse and Logical Form," Lingusitic Inquiry 8, 101139.

Wold, Dag (1996) "Long Distance Selective Binding: The Case of Focus," in Teresa Galloway and Justin Spence (eds.) SALT 6, CLC Publications, Cornell Univ., Ithaca, 311-328.

- (in prep.) Varieties of In Situ Interpretation:_Focal Structure and Scope Assignment at the Syntax/Semantics Interface (working title), M.I.T. Ph.D. thesis. 